\title{
Carbonic anhydrase subunits of the mitochondrial NADH dehydrogenase complex (complex I) in plants
}

\author{
Hans-Peter Braun ${ }^{1}$ and Eduardo Zabaleta ${ }^{2}$
}

${ }^{1}$ Institut für Angewandte Genetik, Universität Hannover, Herrenhäuser Str. 2, D-30419 Hannover, Germany

${ }^{2}$ Instituto de Investigaciones Biológicas, FCEyN, Universidad Nacional de Mar del Plata, Mar del Plata, Argentina.

correspondence:

braun@genetik.uni-hannover.de

e_zabaleta@yahoo.com

\section{$\underline{\text { Content }}$}

1. The respiratory chain of plant mitochondria

2. Carbonic anhydrases

3. Gamma Carbonic Anhydrase subunits of complex I in plants

3.1. Discovery of the $\gamma \mathrm{CA}$ subunits

3.2. Localization of the $\gamma \mathrm{CA}$ subunits

3.3. Characterization of Arabidopsis $\gamma \mathrm{CA}$ knock out mutants

3.4. Activity of the $\gamma \mathrm{CA}$ subunits

4. Possible functional roles of CAs in plant mitochondria

5. Outlook 


\section{Summary}

The mitochondrial NADH dehydrogenase complex (complex I) of plants has a molecular mass of about $1000 \mathrm{kDa}$ and is composed of more than 40 distinct protein subunits. About three quarter of these subunits are homologous to complex I subunits of heterotrophic eukaryotes, whereas the remaining subunits are unique to plants. Among them are three to five structurally-related proteins that resemble an archaebacterial $\gamma$-type carbonic anhydrase $(\gamma \mathrm{CA})$. The $\gamma \mathrm{CA}$ subunits are attached to the membrane arm of complex I on the matrixexposed side and form an extra spherical domain. At the same time, they span the inner mitochondrial membrane and are essential for assembly of the protein complex. Expression of the genes encoding $\gamma \mathrm{CA}$ subunits is reduced if plants are cultivated in the presence of elevated $\mathrm{CO}_{2}$ concentration. The functional role of these subunits within plant mitochondria is currently unknown but might be related to photorespiration. We propose that the complex Iintegrated $\gamma \mathrm{CAs}$ are involved in mitochondrial $\mathrm{HCO}_{3}{ }^{-}$formation to allow efficient recycling of $\mathrm{C}_{\mathrm{i}}$ for $\mathrm{CO}_{2}$ fixation in chloroplasts under high light conditions.

\section{The respiratory chain of plant mitochondria}

Mitochondrial respiration is based on oxidoreductases that transfer electrons from reducing equivalents ( $\mathrm{NADH}$ and $\mathrm{FADH}_{2}$ ) to molecular oxygen. In most eukaryotes four multi-subunit complexes are involved in respiratory electron transport, the NADH dehydrogenase complex (complex I), succinate dehydrogenase (complex II), cytochrome c reductase (complex III) and cytochrome c oxidase (complex IV). Additional so-called "alternative" oxidoreductases occur in some groups of organisms, especially in plants (Siedow and Umbach 1995, Møller 2002, Rasmusson et al. 2004). As a consequence, respiratory electron transport is branched.

However, also the "classical" oxidoreductase complexes of the respiratory chain are quite special in plants because they include several plant-specific subunits. Some of these proteins introduce side activities into the protein complexes of the respiratory chain. For example, the two subunits of the mitochondrial processing peptidase form an integral part of complex III in plants (Braun et al. 1992, Eriksson et al. 1994, Braun et al. 1995). Complex I of plants contains about 10 plant-specific subunits (Heazlewood et al. 2003, Cardol et al. 2004). In fact, the overall molecular mass of plant complex I is clearly larger than the one of mammalian complex I as revealed by direct comparison of these complexes by one-dimensional Blue- 
native PAGE (Jänsch et al. 1995). Three to five of the plant-specific complex I subunits have molecular masses of about $30 \mathrm{kDa}$ and resemble $\gamma$-type carbonic anhydrases (Perales et al. 2004). A 68 kDa subunit represents L-galactono-1,4-lactone dehydrogenase (GLDH), which catalyses the terminal step of mitochondrial ascorbic acid biosynthesis (Millar et al. 2003). However, this protein only forms part of a smaller version of complex I of unknown function (H.P.Braun, unpublished results). Also respiratory complexes II and IV include some plantspecific subunits of unknown function, which most likely introduce side activities into these oxidoreductases (Eubel et al. 2003, Millar et al. 2004). This review summarizes recent results on the carbonic anhydrase subunits of complex I from plants. A hypothesis on the function of these subunits is presented.

\section{Carbonic anhydrases}

Carbonic anhydrases (EC 4.2.1.1) are zinc-containing metalloenzymes that catalyze the interconversion of $\mathrm{CO}_{2}$ and $\mathrm{HCO}_{3}{ }^{-}$. The first enzyme was discovered in human erythrocytes (Meldrum and Roughton, 1933) but meanwhile corresponding activities have been described in many organisms, including animals, plants, eubacteria, and archaebacteria (Hewett-Emmett and Tashian, 1996). CAs play important roles in many physiological processes linked with decarboxylation or carboxylation reactions, e.g. during photosynthesis and respiration. They also participate in transport of inorganic carbon $\left(\mathrm{C}_{\mathrm{i}}\right)$ to actively photosynthesising cells or away from actively respiring cells. CAs probably evolved as enzymes facilitating transmembrane $\mathrm{CO}_{2}$ transport and took on a secondary metabolic role later in metazoan evolution (Henry, 1996). Carbonic anhydrases are encoded by at least five distinct, evolutionarily unrelated gene families. Correspondingly, these enzymes are assigned to four classes designated $\alpha, \beta$ (two different subclasses), $\gamma$, and $\delta$ (So et al., 2004, Sawaya et al., 2006). For this review we mainly focus on $\gamma$-type CA proteins.

So far, only one representative of the $\gamma \mathrm{CA}$ family has been physiologically and biochemically characterized, the $\gamma \mathrm{CA}$ of the archaeon Methanosarcina thermophila ("CAM"; Alber and Ferry, 1994). CAM is a homotrimer composed of proteins in left-handed- $\beta$-helical-fold conformation. Although it is assumed that CAM binds zinc like all other classes of CAs, ironsubstituted forms of the enzyme exhibit the highest $\mathrm{CO}_{2}$ hydration rates. It thus is possible that CAM binds a different co-factor instead of zinc (Tripp et al 2004). High resolution crystal 
structures with bicarbonate bound to the active site of CAM have allowed to predict the residues directly involved in catalysis (Iverson et al. 2000), which meanwhile were confirmed by site-directed mutagenesis (Tripp et al. 2000, Tripp et al. 2002). Three His residues (His 81, 117 and 122) are essential to coordinate the metal ion. $\mathrm{CO}_{2}$ binds adjacent to the zinc-bound hydroxyl group at a position which still is not precisely clear. Solvent-accessible Gln-75, which orients the zinc-bound hydroxide for attack on $\mathrm{CO}_{2}$, is important for $\mathrm{CO}_{2}$ hydration activity. Also Glu-62 is important for the $\mathrm{CO}_{2}$ hydration step, although the specific function is unknown. Glu-84 functions as a proton shuttle residue (PSR). Arg-59 is important for the assembly of monomers into the native trimer. It also is essential for the $\mathrm{CO}_{2}$ hydration step and is postulated to bind bicarbonate. Arg 59 is indirectly hydrogen-bonded to the active site zinc through a network that includes Asp 61, Asp76, His 81, and His 117 (Iverson et al., 2000, Tripp et al., 2002).

There are many open-reading frames in archaea, bacteria and cyanobacteria whose sequences are significantly similar to that of CAM. Most residues important for catalysis are wellconserved in these homologues (the three His coordinating a metal ion, Arg 59, Asp 76 and Gln 75). However, in some cases the active site residues Glu 62 and Glu 84 are not conserved (Iverson et al., 2000, Tripp et al., 2002). To date, none of these homologous proteins has been shown to actually exhibit carbonic anhydrase activity.

\section{Gamma carbonic anhydrase subunits of complex I in plants}

\subsection{Discovery of the $\gamma \mathrm{CA}$ subunits}

Complex I has been characterized for several plants by chromatographic or electrophoretic procedures (Leterme and Boutry 1993, Herz et al. 1994, Rasmusson et al. 1994, Trost et al. 1995, Jänsch et al. 1996, Combettes and Grienenberger 1999). The subunit composition of the purified complex was investigated by SDS-PAGE in combination with direct protein sequencing by cyclic Edman degradation. Some of the obtained N-terminal sequences showed significant similarities to complex I subunits of Neurospora or beef but several others could not be assigned (Leterme and Boutry 1993, Herz et al. 1994). One example is the $29 \mathrm{kDa}$ subunit of complex I from potato and a corresponding $30 \mathrm{kDa}$ subunit of complex I from bean. 
Recently, systematic proteome analyses uncovered several previously unknown mitochondrial proteins in Arabidopsis (Kruft et al. 2001, Millar et al. 2001). Among them, two proteins are homologous to the $29 / 30 \mathrm{kDa}$ subunit of complex I from bean and potato (termed "similar to unknown protein from Rickettsia prowazekii [gene RP516]” in Kruft et al. 2001). Later, proteome analyses based on Blue-native / SDS PAGE revealed that these proteins form part of complex I in Arabidopsis, rice and Chlamydomonas (Heazlewood et al. 2003, Cardol et al. 2004, Perales et al. 2004, Sunderhaus et al. 2006). Arabidopsis complex I was shown to include five structurally related subunits of this type, complex I of rice at least two and complex I of Chlamydomonas three. Thus, small protein families occur for this subunit in all plants investigated. All proteins include hexapeptide repeat (PaaY) motifs and originally have been annotated as "ferripyochelin binding protein-like" (Heazlewood et al. 2003, Cardol et al. 2004) on the basis of sequence similarity with a corresponding protein of Pseudomonas aeruginosa (Sokol, P. et al., University of Calgary, Canada, unpublished results). However, annotation of this prokaryotic protein was recently corrected. It now is annotated as an unknown PaaY containing protein similar to carbonic anhydrases / acetyltransferases of the “isoleucine patch superfamily” (accession number, AAG07140).

Assignment of the plant-specific 29/30 kDa subunits of complex I representing carbonic anhydrases was fist suggested by Parisi et al. (2004). Structural modelling of these proteins revealed a left-handed- $\beta$-parallel $(\mathrm{L} \beta \mathrm{H})$ conformation. Sequence comparisons including more than a hundred homologous sequences of plants showed highest conservation of these proteins to CAM of Methanosarcina termophila, which also belongs to the "isoleucine patch superfamily". The functionally important amino acids His 81, His 117, His 122 (zinc coordination), as well as Arg 59, Asp 61, Gln 75, and Asp 76 are conserved between CAM and most of the complex I subunits of plants. Two other functionally important residues (Glu 62 and Glu 84 of CAM) are missing, but alternative amino acids were identified that may substitute their roles (Parisi et al. 2004). Accordingly, it was proposed that the novel complex I subunits represent a PaaY containing family showing characteristics of $\gamma$ CAs.

Representatives of the novel family are present in plant mitochondria and bacteria but absent in mammals and fungi. Nowadays, this family is designated gamma carbonic anhydrase like family (accession number 51174). Compared to bacteria, $\gamma \mathrm{CAs}$ from plants carry N-terminal extensions, which exhibit the typical properties of mitochondrial targeting sequences. 
The Arabidopsis $\gamma \mathrm{CA}$ protein family is represented by five members. Three of them contain nearly all functionally important amino acids: $\gamma$ CA1 (At1g19580), $\gamma$ CA2 (At1g47260), $\gamma$ CA3 (At5g66510). The two other members are more divergent proteins: $\gamma$ CAL1 (CAL: carbonicanhydrase-like; At5g63510) and $\gamma$ CAL2 (At3g48680). All photosynthetic eukaryotes examined so far contain at least one $\gamma \mathrm{CA}$ and one $\gamma \mathrm{CAL}$ (Perales et al., 2004).

\subsection{Localization of the $\gamma \mathrm{CA}$ subunits}

All five Arabidopsis $\gamma \mathrm{CA} / \gamma \mathrm{CAL}$ subunits were found to be associated with mitochondrial complex I (Heazlewood et al. 2003, Sunderhaus et al. 2006). Mitochondrial localization of the proteins was also shown by in vitro import experiments. Presequences of 3-5 kDa are proteolytically removed after transport in mitochondria is completed (Parisi et al. 2004, Perales et al. 2004). The sub-organellar localization of $\gamma \mathrm{CA}$ and $\gamma \mathrm{CAL}$ proteins was studied by $2 \mathrm{D}$ Blue native/SDS PAGE in combination with immunoblotting using a polyclonal antibody directed against $\gamma \mathrm{CA} 2$ which recognizes all $\gamma \mathrm{CA}$ and $\gamma \mathrm{CAL}$ proteins (Perales et al. 2004). Immune signals were nearly exclusively found in the $30 \mathrm{kDa}$ range of the vertical rows representing complex I and the $\mathrm{I}+\mathrm{III}_{2}$ and $\mathrm{I}_{2}+\mathrm{III}_{4}$ supercomplexes on the $2 \mathrm{D}$ gels. However, some minor immune signals were also visible in the $30 \mathrm{kDa}$ region of smaller protein complexes, which might represent assembly intermediates of complex I or other unknown structures. Further investigations were carried out for the $\gamma \mathrm{CA} 2$ protein using an antibody mono-specific for this protein (Sunderhaus et al., 2006). $\gamma \mathrm{CA} 2$ is exclusively present in the membrane fraction of complex I. Carbonate treatment of isolated mitochondrial membranes did not allow extraction of the protein, indicating a direct anchoring of $\gamma \mathrm{CA} 2$ within the inner mitochondrial membrane.

Direct interaction of CA and CAL proteins was shown by the yeast-two-hybrid system (Perales et al., 2004). $\gamma$ CA proteins are able to form homodimers, but interaction between $\gamma \mathrm{CAL}$ and $\gamma \mathrm{CA}$ proteins is stronger than between $\gamma \mathrm{CA}$ proteins themselves. In contrast, direct interaction between $\gamma \mathrm{CAL}$ proteins could not be monitored using this experimental system. The PaaY domain proved to be absolutely required for stable interaction. Surprisingly, twohybrid screens using $\gamma \mathrm{CA} 2$ allowed to identify only $\gamma \mathrm{CAL}$ proteins but neither complex I 
subunits nor other $\gamma \mathrm{CAs}$. This could be interpreted in favour of a $\gamma \mathrm{CA} / \gamma \mathrm{CAL}$ complex which has to be assembled before its association with complex I (Perales et al., 2004).

Localization of $\gamma \mathrm{CA}$ proteins within complex I was addressed by Blue-native / Blue native PAGE in combination with mass spectrometry (Sunderhaus et al. 2006). Using this gel system, complex I (1000 kDa) becomes divided into two subcomplexes of 600 and $400 \mathrm{kDa}$, which represent the membrane and the matrix arm of this complex. Spots representing these subcomplexes were directly cut out of the gel, trypsinated, and analyzed by mass spectrometry. Four of the five $\gamma \mathrm{CA}$ subunits were identified in the $600-\mathrm{kDa}$ membrane arm and non in the $400 \mathrm{kDa}$ matrix arm (Sunderhaus et al., 2006).

The topological localization of $\gamma \mathrm{CA} 2$ within the membrane arm of complex I was investigated by protease protection experiments using isolated mitoplasts (mitochondria lacking the outer mitochondrial membrane). Comparative immunoblotting analyses of treated and untreated fractions revealed that $\gamma \mathrm{CA} 2$ is protease protected to a large extend (Sunderhaus et al. 2006). However, presence of a $28 \mathrm{kDa}$ degradation product of low abundance indicates that a small part of $\gamma \mathrm{CA} 2$ might be exposed to the mitochondrial intermembrane space. It thus was concluded that the $\gamma \mathrm{CAs}$ from Arabidopsis form part of the membrane arm of complex I and that their $\mathrm{L} \beta \mathrm{H}$ domains point towards the mitochondrial matrix most likely interacting with each other. The precise subunit arrangement of this so called CA domain is presently unknown but deserves further investigations. Interestingly, analysis of Arabidopsis complex I by single particle electron microscopy revealed a spherical extra domain, which is attached to the central part of the membrane arm on its matrix side (Figure 1, Dudkina et al. 2005, Sunderhaus et al. 2006). This domain is absent in complex I particles of all other investigated organisms, except for the alga Polytomella, which is closely related to Chlamydomonas. Thus, presence of CA subunits within complex I correlates with the occurrence of this extra matrix domain, which most likely represents these proteins. The size of this domain would nicely correspond to a $\gamma \mathrm{CA} / \mathrm{CAL}$ trimer like reported for CAM.

\subsection{Characterization of Arabidopsis $\gamma$ CA knock out mutants}

Homozygous Arabidopsis knockout mutants carrying a T-DNA insertion in genes encoding $\gamma \mathrm{CA}$ subunits were generated to investigate their physiological role. Separation of mitochondrial protein complexes by Blue native PAGE or sucrose gradient ultracentrifugation 
revealed drastically reduced complex I levels in a $\gamma c a 2$ mutant and to a lesser extent in $\gamma c a 3$ mutant (Perales et al. 2005). Furthermore, the mitochondrial I + $\mathrm{III}_{2}$ supercomplex was very much reduced in $\gamma c a 2$ mutant plants. Remaining complex I had normal molecular mass and also included the spherical extra domain attached to its membrane arm as described above, suggesting substitution of the $\gamma \mathrm{CA} 2$ subunit by one of the structurally related subunits of $\gamma \mathrm{CA}$ family (Perales et al., 2005, Sunderhaus et al., 2006).

Surprisingly, development of Arabidopsis $\gamma c a$ mutants was normal under standard growth conditions (Perales et al. 2005). However, a suspension cell culture generated from mutant $\gamma \mathrm{CA} 2$ plants exhibited clearly reduced growth rates and respiration. Amounts of singular complex I subunits were reduced, suggesting specific protein degradation or down-regulation of the corresponding nuclear and mitochondrial genes. Abundances of all other protein complexes and alternative oxidoreductases was largely unchanged between mutant and wildtype cells, except for the formate dehydrogenase complex, which was slightly induced, and adrenodoxin (mitochondrial ferredoxin) which was reduced in protein fractions of mutant cells. In summary, comparative characterization of mitochondrial proteins from wild type and $\gamma c a$ cells revealed an important role of $\gamma \mathrm{CA} 2$ for complex I assembly.

\subsection{Activity of $\gamma \mathrm{CA}$ subunits}

Until present, there is no direct physiological evidence of carbonic anhydrase activity of complex I in plants. Several efforts to determine this activity have been performed using different biochemical fractions, including purified Arabidopsis complex I and $\gamma \mathrm{CA}$ proteins overexpressed in E. coli (Eduardo Zabaleta and Hans-Peter Braun, unpublished results). Also, in gel CA activity assays have been carried out using Blue native gels. Possible reasons for these negative results were discussed previously (Perales et al., 2005). However, CA activity of this group of plant-specific complex I subunits is strongly supported by computer modelling. As summarized above, most of the residues important for catalysis are conserved between the prototype $\gamma \mathrm{CA}$ of Methanosarcina thermophila (CAM) and plant $\gamma \mathrm{CAs}$ (Parisi et al., 2004, Perales et al., 2004). Also, antibodies directed against CAM specifically recognize the mitochondrial $\gamma$ CAs (Parisi et al. 2004). One the other side, it currently can not be ruled out that the $\gamma \mathrm{CA} / \gamma \mathrm{CAL}$ subunits of complex I are inactive with respect to CA activity and only bind $\mathrm{CO}_{2}$ and/or bicarbonate in the context of a different physiological process. 
Recently, the crystal structure of a protein of the archaea Pirococcus horikoshii, which is closely related to CAM, was deposited at the Macromolecular Structure Database (MSD) of the European Bioinformatic Institute (EBI) (Jeyakanthan, J. and Tahirov, T.H., http://www.ebi.ac.uk/msd-srv/msdlite/atlas/summary/1v67.html). The structure includes bound $\mathrm{Zn}$ and $\mathrm{HCO}_{3}{ }^{-}$. Since all residues of this protein involved in $\mathrm{Zn}$ - and $\mathrm{HCO}_{3}{ }^{-}$-binding are completely conserved in the $\gamma \mathrm{CA} / \gamma \mathrm{CAL}$ subunits of Arabidopsis complex I, they definitely should be able to bind these two ligands.

EM analysis of complex I revealed a cavity within the membrane arm of complex I on the intermembrane-exposed side directly in opposite to the location of attachment of the extra spherical domain on the matrix side of the membrane arm (Figure 1). It therefore was speculated that the complex I-integrated $\gamma \mathrm{CAs}$ might not only be involved in $\mathrm{CO}_{2}-\mathrm{HCO}_{3}{ }^{-}$ interconversion but at the same time catalyse bicarbonate transport across the inner mitochondrial membrane (Sunderhaus et al., 2006). This transport could be driven by the proton gradient across the inner membrane. If complex I indeed would represent a proton driven bicarbonate translocase, inactivity of the $\gamma \mathrm{CA}$ subunits under in vitro conditions could be due to the absence of the proton gradient necessary for catalysis.

Involvement of complex I integrated $\gamma \mathrm{CA} / \gamma \mathrm{CAL}$ subunits in $\mathrm{CO}_{2}$ metabolisms is also supported by transcriptome analyses for Arabidopsis. Currently, more than 500 Arabidopsis microarray experiments are publicly available at Stanford-Microarray Database (http://genome-www5.stanford.edu/cgi-bin/scriptIndex.pl). Expression of the genes encoding $\gamma \mathrm{CA} 1$ or $\gamma \mathrm{CA} 2$ is very constant under all physiological conditions tested. However, both genes are clearly repressed (>80\%) if Arabidopsis was cultivated in the presence of an elevated $\mathrm{CO}_{2}$ concentration (700 ppm) (Perales et al., 2005). This means that the $\gamma \mathrm{CA}$ subunits of complex I could be especially important if the $\mathrm{CO}_{2}$ concentration is low. Indirect evidence for involvement of $\gamma \mathrm{CA} 2$ in mitochondrial one-carbon metabolism also comes from the observation that formate dehydrogenase is up-regulated in the $\gamma c a 2$ mutant line. The fact that upregulation of this protein has not been observed in mutants lacking other complex I subunits (Sabar et al., 2000, Pineau et al., 2005) indicates a specific relationship between the plant $\gamma \mathrm{CA}$ proteins and one-carbon metabolism. 


\section{Possible functional roles of $\gamma \mathrm{CAs}$ in plant mitochondria}

What could be the physiological role of carbonic anhydrases in mitochondria? Animal cells are known to have several $\alpha$-type carbonic anhydrases, two of which are localized in mitochondria (carbonic anhydrase VA and VB, reviewed in Nishimori et al., 2005). These $\alpha \mathrm{CAs}$ are of low abundance. VA was first discovered and only is present in hepatocytes (Dogson and Foster, 1984). It is speculated to be involved in maintenance of bicarbonate production for carboxylation reactions of several important biosynthetic pathways, such as lipogenesis, gluconeogenesis, and ureagenesis, among others (Dogson and Forster, 1986). VB has a much wider tissue distribution (Shah et al., 2000), suggesting a different physiological role. However, bicarbonate production in mitochondria for the above mentioned biosynthetic pathways most likely is not important in plants, because the pathways do not occur in plant mitochondria. Therefore, the complex I integrated $\gamma$ CAs of plants must have a different role.

Also in Chlamydomonas, two mitochondrial carbonic anhydrases were described (Eriksson et al., 1996). They belong to the $\beta C A$ family and are distinct from the much later discovered $\gamma$ CAs present within complex I of this organism (Cardol et al. 2004). Both $\beta$ CAs are encoded by two almost identical nuclear genes which are expressed in the light at low external $\mathrm{CO}_{2}$ concentrations (Villand et al., 1997; Eriksson et al., 1998). Like in the case of the complex Iintegrated $\gamma$ CAs, activity of the $\beta$ CAs, which most likely are localized within the mitochondrial matrix, so far could not be monitored. Different hypothesis were suggested concerning their function: (i) $\mathrm{CO}_{2}-\mathrm{HCO}_{3}{ }^{-}$interconversion by the $\beta \mathrm{CAs}$ might be important for buffering matrix $\mathrm{H}^{+}$concentration upon initiation of photorespiration when cells are transferred from high to low $\mathrm{CO}_{2}$ conditions (Eriksson et al., 1996). However, it was shown that the $\beta C A$ genes are also expressed at high $\mathrm{CO}_{2}$ concentrations $(0.2 \%$ [v/v] in air), if sufficient $\mathrm{NH}_{4}{ }^{+}$(1 to $10 \mathrm{mM}$ ) is available (Giordano et al., 2003). (ii) $\mathrm{HCO}_{3}{ }^{-}$formation by the mitochondrial $\beta C A$ As might be important for anaplerotic reactions which require inorganic carbon to build up C4 compounds for the TCA cycle via $\beta$-carboxylations (Giordano et al., 2003). The provision of inorganic carbon for these reactions could be crucial to sustain amino acid and protein synthesis. (iii) $\mathrm{HCO}_{3}{ }^{-}$formation possibly is important for limiting loss of $\mathrm{CO}_{2}$ caused by photorespiration (Raven 2001). Prerequisite for this hypothesis is the presence of a bicarbonate transporter within the mitochondrial membranes, which so far has not been described. In contrast to $\mathrm{CO}_{2}, \mathrm{HCO}_{3}{ }^{-}$could be transported actively from mitochondria to the cytoplasm and afterwards into plastids, allowing recycling of excess of mitochondrial $\mathrm{CO}_{2}$ for 
carbon-fixation by RubisCO. Alternatively it was suggested that the exported $\mathrm{HCO}_{3}{ }^{-}$might be used for $\mathrm{NH}_{4}{ }^{+}$fixation (Raven 2001). Indeed, expression of the $\beta C A s$ from Chlamydomonas was shown to be modulated by $\mathrm{NH}_{4}{ }^{+}$supply as well as $\mathrm{CO}_{2}$ supply (Giordano et al., 2003)

The Arabidopsis genome includes several genes encoding proteins of the $\alpha$ - and $\beta C A$ families (Moroney et al. 2001). Targeting prediction analyses using the deduced protein sequences does not indicate presence of $\alpha \mathrm{CAs}$ within mitochondria. In contrast, one of the six $\beta C A s$ encoded by the Arabidopsis mitochondrial genome (At1g58180) is predicted to have mitochondrial localization using several different targeting prediction software tools. However, this protein never was detected in mitochondrial fractions by proteome analyses and therefore either has a different subcellular location or is of very low abundance. In consequence, the five $\gamma \mathrm{CA} / \gamma \mathrm{CAL}$ proteins of complex I seem to represent the only mitochondrial carbonic anhydrases in higher plants.

Interesting insights into the functional role of CAs come from investigations with cyanobacteria, which might be helpful to better understand the role of the complex Iintegrated CAs in plants. Also in cyanobacteria, involvement of a complex I-like enzyme (termed NDH-1 complex in cyanobacteria and plastids of higher plants) in carbon dioxide metabolism was reported, which is important in the context of a carbon dioxide concentration mechanism (reviewed by Badger and Price, 2003). NAD(P)H dehydrogenase type 1 (NDH-1) complexes are known to have multiple functions in both cyanobacteria and higher plants. Common for both groups of organisms is a function of the NDH-1 complexes in respiration (chlororespiration in chloroplasts) and in cyclic electron transport around photosystem I (PSI) (Munekage et al., 2004). A number of distinct types of NDH-1 complexes were described for $\beta$-cyanobacteria, which were found to have different physiological roles: a conventional respiratory NDH-1 complex (NDH-I/1/2, or NDH-1L and NDH-1M, Battchikova et al., 2005) and two specialized NAD-1 complexes termed NDH-13 (also designated NDH-1S) and NDH$1_{4}$ complexes involved in a $\mathrm{CO}_{2}$ uptake mechanism. The latter two complexes include socalled Chp Y/Cup A and ChpX/Cup B polypeptides. The Chp proteins are integral membrane proteins directly catalysing conversion of $\mathrm{CO}_{2}$ into $\mathrm{HCO}_{3}{ }^{-}$, which is linked to electron transfer and proton translocation within the NDH-1 $1_{3 / 4}$ complexes (Maeda et al., 2002, Herranen et al., 2004, Zhang et al., 2005). Although Chp proteins do not exhibit sequence homologies to any member of the known CA protein families, two conserved histidine residues and one conserved cysteine residue are believed to allow coordination of $\mathrm{Zn}$ (Battchikova et al. 2005). 
The Chp proteins possibly should be defined as an additional independent class of carbonic anhydrases.

By analogy, the complex I-integrated $\gamma \mathrm{CAs}$ of plant mitochondria might also catalyse a proton driven interconversion of $\mathrm{CO}_{2}$ and $\mathrm{HCO}_{3}{ }^{-}$as discussed above. However, in contrast to cyanobacteria, which use this mechanisms to actively increase their internal $\mathrm{C}_{\mathrm{i}}$ concentration, plant mitochondria rather have a problem with excess of $\mathrm{CO}_{2}$ due to catabolic reactions within the mitochondrial matrix, especially during photorespiration. Interestingly, subunits of an $\mathrm{NDH}-1_{3}$ like complex were recently also described for thylakoid membranes of higher plants, whose abundance increases under conditions of $C_{i}$ limitation (Herranen et al., 2004, Zhang et al., 2005, reviewed in Badger et al., 2006). However, involvement of these proteins in $\mathrm{CO}_{2}-$ $\mathrm{HCO}_{3}{ }^{-}$interconversion has not been shown.

In general, research with cyanobacteria points to a linkage of $\mathrm{CO}_{2}$ hydration at the plasmamembrane and proton translocation across this membrane (Badger and Price, 2003, Badger et al., 2006). It was suggested that electron transport-dependent proton translocation across membranes causes formation of local alkaline pockets (Kaplan and Reinhold, 1999). Since the equilibrium of $\mathrm{CO}_{2}-\mathrm{HCO}_{3}{ }^{-}$interconversion shifts very much to the $\mathrm{HCO}_{3}{ }^{-}$side at $\mathrm{pH}>8$, the alkaline pockets are an ideal micro-location for CAs involved in $\mathrm{HCO}_{3}{ }^{-}$ generation. This might be the reason for the presence of complex I-integrated CA activities in cyanobacteria and plant mitochondria, which most likely arose by convergent evolution.

Cyanobacteria also contain a protein homologous to $\gamma$-type carbonic anhydrases. This protein forms part of the carboxysome and is termed $\mathrm{CcmM}$ (product of the ccmM gene; $\mathrm{ccm}$ : $\mathrm{CO}_{2}$ concentrationg mechanisms). It so far was not shown to have CA activity. The protein comprises 539 amino acids and has a bipartite structure, which resembles CAM within its Nterminal and the small subunit of RubisCO within its C-terminal half. Its role in $\mathrm{CO}_{2}$ metabolism is so far unclear. Disruption of the $c c m M$ gene in the cyanobacterium Synechocystis sp. PCC 6803 causes carboxysome deficiency and impaired growth at ambient $\mathrm{CO}_{2}$ conditions (Ludwig et al., 2000, Berry et al., 2005). Physiological characterization of this protein might give new insights into the functional role of the mitochondrial $\gamma \mathrm{CA}$ protein family. 
In summary, although direct evidence for CA activity of the $\gamma \mathrm{CA} / \gamma \mathrm{CAL}$ subunits of mitochondrial complex I in plants is still lacking, data from a large number of sources discussed above indicate that these proteins are involved in $\mathrm{CO}_{2}$ metabolism, most likely represent carbonic anhydrases and possibly also are involved in proton-driven bicarbonate transfer across the inner mitochondrial membrane. Down-regulation of the $\gamma \mathrm{CA}$ genes upon cultivation of Arabidopsis in the presence of elevated $\mathrm{CO}_{2}$ concentration, which is known to repress photorespiration, points to a role of the $\gamma \mathrm{CA}$ subunits in the context of this metabolic pathway. We therefore suggest involvement of the $\gamma \mathrm{CA}$ subunits in $\mathrm{HCO}_{3}{ }^{-}$formation to allow efficient recycling of mitochondrial $\mathrm{C}_{\mathrm{i}}$ for $\mathrm{CO}_{2}$ fixation in chloroplasts (Figure 2) in accordance with the hypothesis of Raven (2001).

\section{Outlook}

So far, many aspects of $\gamma \mathrm{CA} / \gamma \mathrm{CAL}$ function are still a mystery. Further experiments on physiological and genetic levels will be required to better understand the biological role of this interesting group of proteins. Since homologues of the plant mitochondrial $\gamma \mathrm{CA} / \gamma \mathrm{CAL}$ proteins are present in cyanobacteria and other eubacteria, gene knock out approaches using prokaryotes might be the fastest way to obtain new insights. But the biological context of the mitochondrial $\gamma \mathrm{CAs} / \gamma \mathrm{CALs}$ of green algae and higher plants might be different. Knock out experiments using Arabidopsis so far did not allow to clarify the physiological role of these proteins, probably due to functional redundancies within this protein family. Characterization of double and triple knock out mutants is on the way in our laboratories. However, presence of five structurally related $\gamma \mathrm{CA}$ or $\gamma \mathrm{CAL}$ proteins in Arabidopsis probably also reflects distinct functional roles of the individual members of this protein family. Expression analyses of the corresponding genes in different tissues and developmental stages will be important to investigate this aspect. Data already available in Arabidopsis microarray databases might be helpful in this respect.

The complex I-integrated $\gamma \mathrm{CA} / \gamma \mathrm{CAL}$ subunits are another fascinating example underlining the uniqueness of plant mitochondria. 


\section{Acknowledgements}

We thank Jesco Heinemeyer for critically reading of the manuscript. Research in our Laboratories is supported by the Deutsche Forschungsgemeinschaft (DFG) and the Agencia Nacional de Promoción Científica y Tecnológica (ANPCyT), Argentina.

\section{References}

Alber BE, Ferry JG. (1994) A carbonic anhydrase from the archaeon Methanosarcina thermophila. Proc Natl Acad Sci U S A. 91: 6909-6913

Badger MR, Price GD. (2003) $\mathrm{CO}_{2}$ concentrating mechanisms in cyanobacteria: molecular components, their diversity and evolution. J Exp Bot. 2003 54: 609-622.

Badger MR, Price GD, Long BM, Woodger FJ. (2006) The environmental plasticity and ecological genomics of the cyanobacterial $\mathrm{CO}_{2}$ concentrating mechanism. J Exp Bot. 57: 249-265

Battchikova N, Zhang P, Rudd S, Ogawa T, Aro EM. (2005) Identification of NdhL and Ss11690 (NdhO) in NDH-1L and NDH-1M complexes of Synechocystis sp. PCC 6803. J Biol Chem.280: 2587-2595

Berry S, Fischer JH, Kruip J, Hauser M, Wildner GF (2005) Monitoring cytosolic pH of carboxysomedeficient cells of Synechocystis sp. PCC 6803 using fluorescence analysis. Plant Biol 7: 342-347

Braun HP, Emmermann M, Kruft V, Schmitz UK (1992) The general mitochondrial processing peptidase from potato is an integral part of cytochrome c reductase of the respiratory chain. EMBO J 11: 3219-3227

Braun HP, Emmermann M, Kruft V, Bödicker M, Schmitz UK (1995) The general mitochondrial processing peptidase from wheat is integrated into the cytochrome $b_{1} c_{1}$ complex of the respiratory chain. Planta 195: 396-402

Cardol P, Vanrobaeys F, Devreese B, Van Beeumen J, Matagne RF, Remacle C. (2004) Higher plantlike subunit composition of mitochondrial complex I from Chlamydomonas reinhardtii: 31 conserved components among eukaryotes. Biochim Biophys Acta. 1658: 212-224

Combettes B, Grienenberger JM (1999) Analysis of wheat mitochondrial complex I purified by a onestep immunoaffinity chromatography. Biochemie $81:$ 645-653

Dodgson SJ, Forster RE 2 ${ }^{\text {nd }}$, Storey BT (1984) The role of carbonic anhydrase in hepatocyte metabolism. Ann N Y Acad Sci 429: 516-524

Dodgson SJ, Forster RE $2^{\text {nd }}$ (1986) Inhibition of CA V decreases glucose synthesis from pyruvate. Arch Biochem Biophys 1986 251: 198-204

Dudkina NV, Eubel H, Keegstra W, Boekema EJ, Braun HP (2005) Structure of a mitochondrial supercomplex formed by respiratory-chain complexes I and III. Proc Natl Acad Sci USA 102: 3225-3229

Eriksson AC, Sjöling S, Glaser E (1994) The ubiquinol cytochrome c oxidoreductase complex of spinach leaf mitochondria is involved in both respiration and protein processing. Biochim Biophys Acta 1186: 221-231 
Eriksson M, Karlsson J, Ramazanov Z, Gardestrom P, Samuelsson G. (1996) Discovery of an algal mitochondrial carbonic anhydrase: molecular cloning and characterization of a low- $\mathrm{CO}_{2}$-induced polypeptide in Chlamydomonas reinhardtii. Proc Natl Acad Sci USA 93: 12031-12034

Eriksson M, Villand P, Gardestrom P, Samuelsson G (1998) Induction and Regulation of Expression of a Low- $\mathrm{CO}_{2}$-Induced Mitochondrial Carbonic Anhydrase in Chlamydomonas reinhardtii. Plant Physiol. 116: 637-641.

Eubel H, Jänsch L, Braun HP (2003) New insights into the respiratory chain of plant mitochondria: supercomplexes and a unique composition of complex II. Plant Physiol 133: 274-286

Giordano M, Norici A, Forssen M, Eriksson M, Raven JA (2003) An anaplerotic role for mitochondrial carbonic anhydrase in Chlamydomonas reinhardtii. Plant Physiol 132: 2126-2134

Gray MW, Burger G, Lang BF (2001) The origin and early evolution of mitochondria. Genome Biol 2: REVIEWS1018

Heazlewood JL, Howell KA, Millar AH. (2003) Mitochondrial complex I from Arabidopsis and rice: orthologs of mammalian and fungal components coupled with plant-specific subunits. Biochim Biophys Acta 1604: 159-169

Henry RP (1996) Multiple roles of carbonic anhydrase in cellular transport and metabolism. Annu Rev Physiol. 58: 523-538

Herranen M, Battchikova N, Zhang P, Graf A, Sirpio S, Paakkarinen V, Aro EM (2004) Towards functional proteomics of membrane protein complexes in Synechocystis sp. PCC 6803. Plant Physiol 134: 470-481

Herz U, Schroder W, Liddell A, Leaver CJ, Brennicke A, Grohmann L (1994) Purification of the NADH:ubiquinone oxidoreductase (complex I) of the respiratory chain from the inner mitochondrial membrane of Solanum tuberosum. J Biol Chem 269: 2263-2269

Hewett-Emmett D, Tashian RE (1996) Functional diversity, conservation, and convergence in the evolution of the alpha-, beta-, and gamma-carbonic anhydrase gene families. Mol Phylogenet Evol 5: 50-77

Iverson TM, Alber BE, Kisker C, Ferry JG, Rees DC (2000) A closer look at the active site of gammaclass carbonic anhydrases: high-resolution crystallographic studies of the carbonic anhydrase from Methanosarcina thermophila. Biochemistry 39: 9222-9231

Jänsch L, Kruft V, Schmitz UK and Braun HP (1995) Cytochrome c reductase from potato does not comprise three core proteins but contains an additional low molecular weight subunit. Eur J Biochem 228: 878-885

Jänsch L, Kruft V, Schmitz UK, Braun HP (1996) New insights into the composition, molecular mass and stoichiometry of the protein complexes of plant mitochondria. Plant J 9: 357-368

Kaplan A and Reinbold L (1999) $\mathrm{CO}_{2}$ concentrating mechanisms in photosynthetic microorganisms. Ann Rev Plant Physiol Plant Mol Biol 50: 539-570

Kruft V, Eubel H, Jansch L, Werhahn W, Braun HP (2001) Proteomic approach to identify novel mitochondrial proteins in Arabidopsis. Plant Physiol 127: 1694-1710

Leterme S, Boutry M (1993) Purification and preliminary characterization of mitochondrial complex I (NADH: ubiquinone reductase) from broad bean (Vicia faba L.). Plant Physiol 102: 435-443 
Ludwig M, Sültemeyer D, Price GD (2000) Isolation of ccmKLMN genes from the marine cyanobacterium, Synechoccus PCC7002 (Cyanophceae), and evidence that CcmM is essential for carboxysome assembly. J Phycology 36: 1109-1118

Maeda S, Badger MR, Price GD (2002) Novel gene products associated with NdhD3/D4-containing NDH-1 complexes are involved in photosynthetic $\mathrm{CO}_{2}$ hydration in the cyanobacterium, Synechococcus sp. PCC7942. Mol Microbiol 43: 425-435

Meldrum NU and Roughton FJ (1933) Carbonic anhydrase: its preparation and properties. J Physiol 80: $113-141$

Millar AH, Sweetlove LJ, Giege P, Leaver CJ. (2001) Analysis of the Arabidopsis mitochondrial proteome. Plant Physiol 127: 1711-1727

Millar AH, Mittova V, Kiddle G, Heazlewood JL, Bartoli CG, Theodoulou FL and Foyer CH (2003) Control of ascorbate synthesis by respiration and its implications for stress responses. Plant Physiol 133, 443-447

Millar AH, Eubel H, Jänsch L, Kruft V, Heazlewood L, Braun HP (2004) Mitochondrial cytochrome c oxidase and succinate dehydrogenase contain plant-specific subunits. Plant Mol Biol 56: 77-89

Møller IM (2002) A new dawn for plant mitochondrial NAD(P)H dehydrogenases. Trends Plant Sci 7: 235-237

Moroney JV, Bartlett SG, Samuelsson G (2001) Carbonic Anhydrases in plants and algae. Plant \& Cell Physiol 24: 141-153

Munekage Y, Hashimoto M, Miyake C, Tomizawa K, Endo T, Tasaka M, Shikanai T (2004) Cyclic electron flow around photosystem I is essential for photosynthesis. Nature 429: 579-582

Nishimori I, Vullo D, Innocenti A, Scozzafava A, Mastrolorenzo A, Supuran CT (2005) Carbonic anhydrase inhibitors. The mitochondrial isozyme VB as a new target for sulfonamide and sulfamate inhibitors. J Med Chem. 48: 7860-7866.

Parisi G, Perales M, Fornasari MS, Colaneri A, Gonzalez-Schain N, Gomez-Casati D, Zimmermann S, Brennicke A, Araya A, Ferry JG, Echave J, Zabaleta E (2004) Gamma carbonic anhydrases in plant mitochondria. Plant Mol Biol 55: 193-207

Perales M, Parisi G, Fornasari MS, Colaneri A, Villarreal F, Gonzalez-Schain N, Echave J, GomezCasati D, Braun HP, Araya A, Zabaleta E (2004) Gamma carbonic anhydrase like complex interact with plant mitochondrial complex I. Plant Mol Biol 56: 947-957

Perales M, Eubel H, Heinemeyer J, Colaneri A, Zabaleta E, Braun HP (2005) Disruption of a nuclear gene encoding a mitochondrial gamma carbonic anhydrase reduces complex I and supercomplex I+III 2 levels and alters mitochondrial physiology in Arabidopsis. J Mol Biol 350: 263-277.

Pineau B, Mathieu C, Gerard-Hirne C, De Paepe R, Chetrit P (2005) Targeting the NAD7 subunit to mitochondria restores a functional complex I and a wild type phenotype in the Nicotiana sylvestris CMS II mutant lacking nad7. J Biol Chem. 280: 25994-26001

Rasmusson AG, Mendel-Hartvig J, Moller IM, Wiskich JT (1994) Isolation of the rotenone-sensitive NADH-ubiquinone reductase (complex I) from red beet mitochondria. Physiol Plant 90: 607615

Rasmusson AG, Soole KL, Elthon TE (2004) Alternative NAD(P)H dehydrogenases of plant mitochondria. Annu Rev Plant Biol 55: 23-39 
Raven JA (2001) A role for mitochondrial carbonic anhydrase in limiting $\mathrm{CO}_{2}$ leakage from low $\mathrm{CO}_{2-}$ grown cells of Chlamydomonas reinhardtii. Plant Cell Environ 24: 261-265

Sabar M, De Paepe R, de Kouchkovsky Y (2000) Complex I impairment, respiratory compensations, and photosynthetic decrease in nuclear and mitochondrial male sterile mutants of Nicotiana sylvestris. Plant Physiol 124: 1239-1250

Sawaya MR, Cannon GC, Heinhorst S, Tanaka S, Williams EB, Yeates TO, Kerfeld CA (2006) The structure of beta -carbonic anhydrase from the carboxysomal shell reveals a distinct subclass with one active site for the price of two. J Biol Chem, in press

Siedow LN and Umbach AL (1995) Plant mitochondrial electron transfer and molecular biology. Plant Cell 7: 821-831

Shah GN, Hewett-Emmett D, Grubb JH, Migas MC, Fleming RE, Waheed A, Sly WS (2000) Mitochondrial carbonic anhydrase CA VB: differences in tissue distribution and pattern of evolution from those of CA VA suggest distinct physiological roles. Proc Natl Acad Sci USA 97: 1677-1682

So AK, Espie GS, Williams EB, Shively JM, Heinhorst S, Cannon GC (2004) A novel evolutionary lineage of carbonic anhydrase (epsilon class) is a component of the carboxysome shell. $\mathrm{J}$ Bacteriol 186: 623-630

Sunderhaus S, Dudkina N, Jansch L, Klodmann J, Heinemeyer J, Perales M, Zabaleta E, Boekema E, Braun HP (2006) Carbonic anhydrase subunits form a matrix-exposed domain attached to the membrane arm of mitochondrial complex I in plants. J Biol Chem 281: 6482-6488

Tripp BC, Ferry JG. (2000) A structure-function study of a proton transport pathway in the gammaclass carbonic anhydrase from Methanosarcina thermophila. Biochemistry 39: 9232-9240

Tripp BC, Tu C, Ferry JG (2002) Role of arginine 59 in the gamma-class carbonic anhydrases. Biochemistry 41: 669-678

Tripp BC, Bell CB 3rd, Cruz F, Krebs C, Ferry JG (2004) A role for iron in an ancient carbonic anhydrase. J Biol Chem 279: 6683-6687

Trost P, Bonora P, Scagliarini S, Pupillo P (1995) Purification and properties of NAD(P)H: (quinoneacceptor) oxidoreductase of sugarbeet cells. Eur J Biochem 234: 452-458

Villand P, Eriksson M, Samuelsson G (1997) Carbon dioxide and light regulation of promoters controlling the expression of mitochondrial carbonic anhydrase in Chlamydomonas reinhardtii. Biochem J 327: 51-57

Zhang P, Battchikova N, Paakkarinen V, Katoh H, Iwai M, Ikeuchi M, Pakrasi HB, Ogawa T, Aro EM (2005) Isolation, subunit composition and interaction of the NDH-1 complexes from Thermosynechococcus elongatus BP-1. Biochem J 390: 513-520 


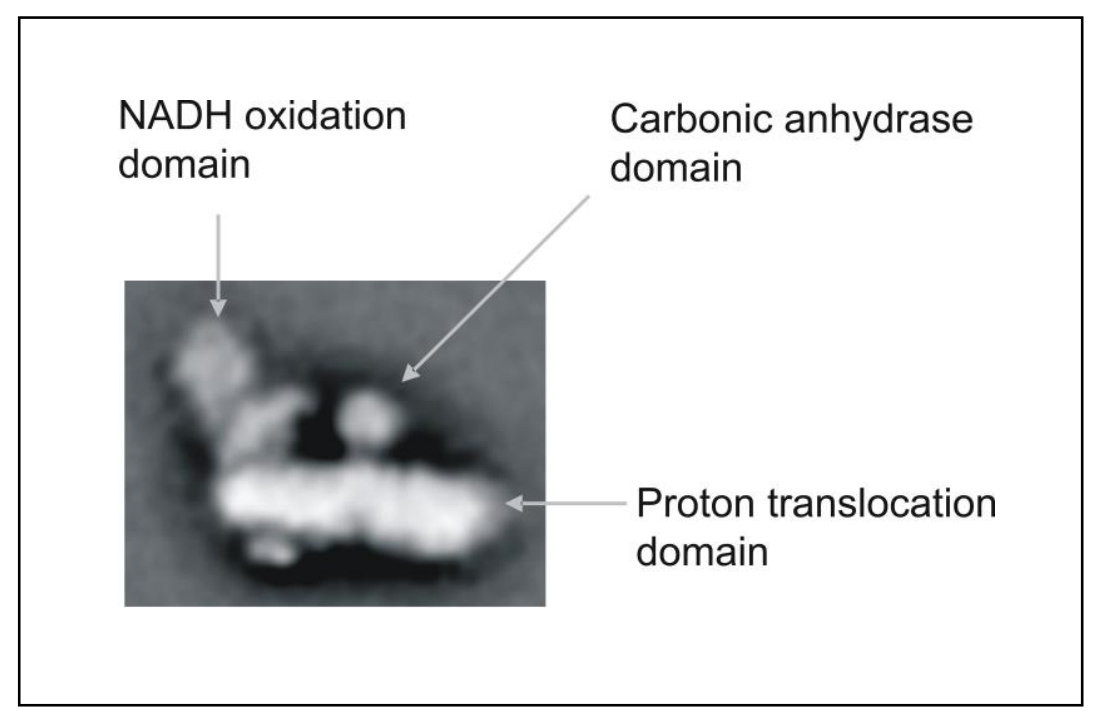

Figure 1: Structure of complex I from Arabidopsis (from: Sunderhaus et al. 2006, J. Biol. Chem. 281, 6482-6488, with permission). The carbonic anhydrase subunits form a characteristic extra domain, which protrudes into the mitochondrial matrix.

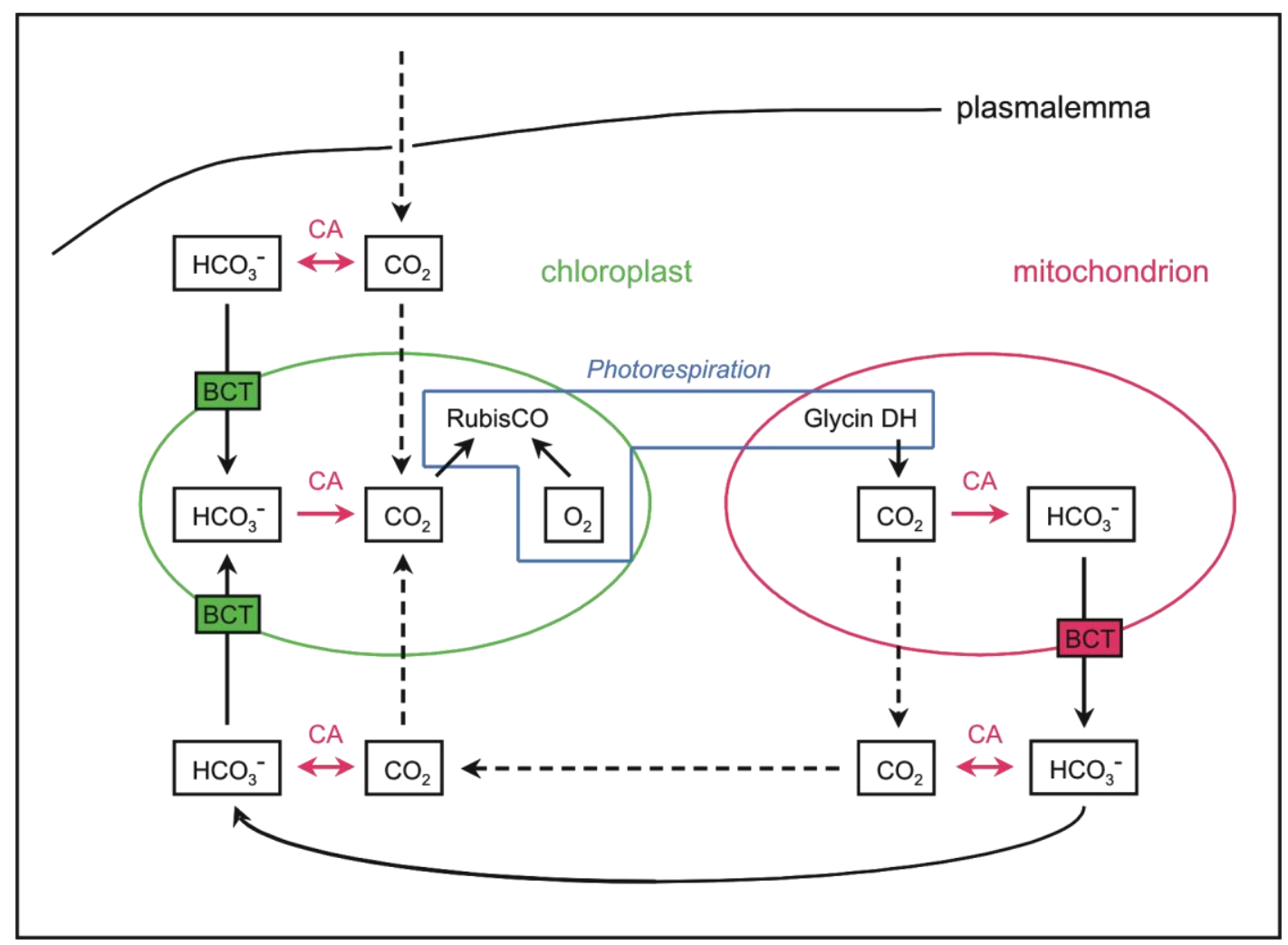

Figure 2: Model for the function of the complex I-integrated carbonic anhydrases in plants. Under high light conditions, $\mathrm{CO}_{2}$ concentration decreases in chloroplasts due to RubisCO activity. At the same time, $\mathrm{CO}_{2}$ concentration increases in mitochondria due to the oxygenation side-activity of RubisCO in combination with the decarboxylation activity of the mitochondrial glycine dehydrogenase (Photorespiration). Equilibration of the $\mathrm{CO}_{2}$ imbalance between mitochondria and plastids could be based on diffusion of $\mathrm{CO}_{2}$ across organellar membranes (slow, dashed line) or by active transport of $\mathrm{HCO}_{3}{ }^{-}$by bicarbonate transporters (BCTs) (fast, solid line). The mitochondrial CAs are proposed to play a role in the latter mechanisms. 